\title{
Imatinib-Induced Interstitial Pneumonitis Successfully Switched to Nilotinib in a Patient with Prior History of Mycobacterium tuberculosis Infection
}

\author{
Mycobacterium tuberculosis Enfeksiyonu Öyküsü Olan Hastada Başarılı Bir Şekilde \\ Nilotinibe Geçilen Imatinib ile Uyarılmış Interstisyel Pnömonitis
}

\author{
Zhuan-Bo Luo ${ }^{1}$, Ning Xu' ${ }^{1}$, Xiao-Ping Huang ${ }^{1}$, Gui-fang Ouyang ${ }^{2}$ \\ ${ }^{1}$ Department of Respiratory Diseases, Ningbo First Hospital, Affiliated Medical School of Ningbo University, Ningbo, China \\ ${ }^{2}$ Department of Hematology, Ningbo First Hospital, Affiliated Medical School of Ningbo University, Ningbo, China
}

To the Editor,

Imatinib mesylate (IM) has been proven to be an effective treatment of chronic myeloid leukemia (CML) and this drug is well tolerated [1]. Interstitial lung disease (ILD) associated with imatinib therapy is rare. We report the case of a patient who had a prior treatment history of Mycobacterium tuberculosis infection and developed interstitial pneumonia after 10 months of imatinib for CML and who has not relapsed since the introduction of the recent tyrosine kinase inhibitor nilotinib.

A 48-year-old Chinese man was diagnosed with chronic-phase Philadelphia chromosome-positive CML in January 2015. His medical history was unremarkable, but he had a history of previous treatment for pulmonary tuberculosis 25 years ago. He was initially treated with IM at a dose of $400 \mathrm{mg}$ daily, which was well tolerated. Complete hematological response was rapidly achieved after 2 months. Following the administration of imatinib, the patient gradually developed a dry cough and dyspnea on exertion. In November 2015, he visited the clinic because of progressing nonproductive cough. He had been treated with imatinib at a dose of $400 \mathrm{mg} /$ day for 10 months. On examination, fine crackles were audible, predominantly in both posterior lower lung fields. No elevations of the acutephase reactants were detected, and the immunoglobulin E blood level was within the normal limits. Rheumatoid factor was negative, and antinuclear antibodies were positive at 1/100 with homogeneous staining. Sputum culture was negative and no acid-fast bacilli were observed. Lung function estimation demonstrated mild impairment of gas exchange with diffusing capacity [carbon monoxide diffusion in the lung (DLCO)] of $5.61 \mathrm{mmol} / \mathrm{min}$ per $\mathrm{kPa}(51.9 \%$ predicted) and mild restrictive impairment with forced vital capacity of $3.30 \mathrm{~L}$ (69.1\% predicted). A chest radiograph showed fibrotic scar lesions in the left upper lung field associated with the previous pulmonary tuberculosis. A computed tomography scan (Figure 1A) showed significant extension of the interstitial lung abnormalities, predominantly in the lower lobes. Bronchoscopy revealed normal airways, and histopathological analysis of the transbronchial lung biopsy demonstrated nonspecific interstitial pneumonitis, showing thickened alveolar septa with modest infiltration of chronic inflammatory cells and slight interstitial fibrosis (Figures $2 \mathrm{~A}$ and $2 B$ ). As these findings were highly suggestive of imatinib-induced interstitial pneumonitis, this agent was discontinued and was replaced by nilotinib. At the same time, prednisone at $30 \mathrm{mg} /$ day was given during the initial days, and it was slowly tapered to $10 \mathrm{mg} /$ day over 2 months. Because no signs of recurrence of pulmonary tuberculosis were detected and the patient was afraid of the side effects of anti-tuberculosis drugs, we did not give anti-tuberculosis prophylaxis, but we maintained close follow-up. This resulted in a gradual improvement in his clinical condition. Partial radiological resolution was observed after 4 months and further improved at 8 months (Figure 1B). DLCO improved to $6.78 \mathrm{mmol} / \mathrm{min}$ per $\mathrm{kPa}(62.8 \%$ predicted) and forced vital capacity was $4.50 \mathrm{~L}(83.3 \%$ predicted). The switch to nilotinib at $800 \mathrm{mg}$ daily was well tolerated and followed by complete cytogenetic and major molecular response sustained for 8 months.

IM is a targeted therapy that is highly active in patients with CML. It acts by inhibition of tyrosine kinase of the BCR-ABL fusion oncoprotein specific to CML. ILD is a rare adverse event associated with IM therapy. Few case series have been reported $[2,3,4,5]$. In the present case, the diagnosis of IM-induced ILD was made based on history, clinical symptoms, radiological findings, and pathological results. Furthermore, other etiologic factors for ILD were excluded via microbiologic and clinical studies.

Until recently, there has been a lack of data for specific risk factors for the development of IM-induced ILD. However, the incidence of the disease seems higher in patients with preexisting 


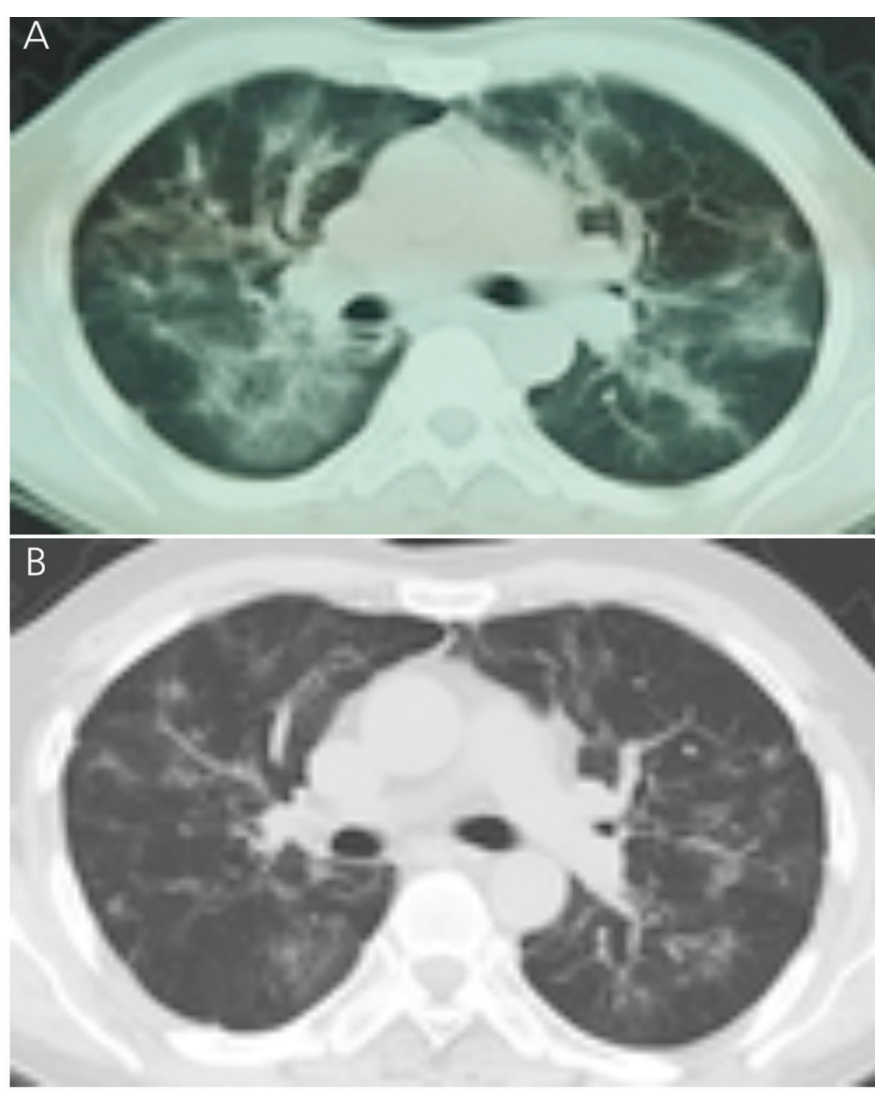

Figure 1. A) Chest computed tomography (CT) shows bilateral diffuse subpleural nodules, interlobular septal thickening, reticulation, and peribronchial ground glass opacities in both lungs. B) Chest CT scan 8 months after the switch to prednisone and nilotinib: lung abnormalities were decreased.

pulmonary diseases. The largest case series study from Japan, which analyzed 27 patients with IM-induced ILD [6], revealed that preexisting lung disease was present in more than $40 \%$ of patients with IM-induced ILD. In this case, we had found fibrotic change and pleural thickening of the left upper lung associated with prior infection of Mycobacterium tuberculosis. Thus, the case raises the possibility of the association between IM-induced ILD and airway injury related to prior infection of Mycobacterium tuberculosis. Physicians caring for a patient presenting with respiratory symptoms while on imatinib therapy should consider interstitial pneumonitis, especially in patients with previous lung or airway injuries resulting from prior infection of Mycobacterium tuberculosis.

Among the other tyrosine kinase inhibitors, similar pulmonary complications were reported with dasatinib [7] and gefitinib [8] in Japan, but not with nilotinib. In this case, 8 months after the introduction of nilotinib, interstitial pneumonitis had not recurred. Although the mechanistic basis for the absence of cross-intolerance is not fully understood, second-generation nilotinib appears to be an option in cases of ILD induced by other tyrosine kinase inhibitors.
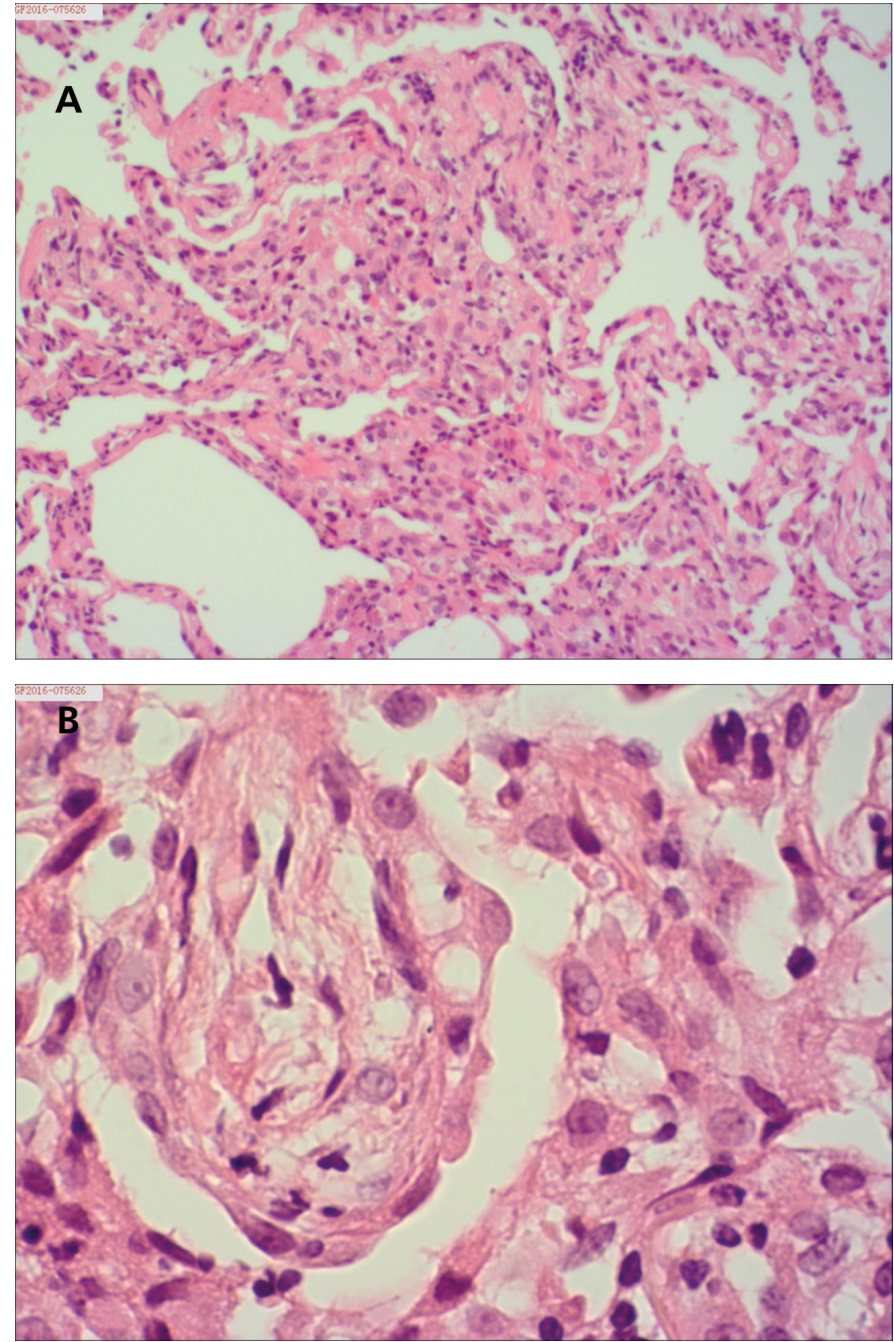

Figure 2. A and B) Histopathological appearance of transbronchial lung biopsy specimens. Thickened alveolar septa with modest infiltration of chronic inflammatory cells and slight interstitial fibrosis are observed. Hematoxylin and eosin stain (H\&E), 100 (A); H\&E stain, 400× (B).

Keywords: Imatinib mesylate, Interstitial pneumonitis, Chronic myeloid leukemia, Nilotinib, Tuberculosis

Anahtar Sözcükler: İmatinib mesilat, İnterstisyel pnömonitis, Kronik miyeloid lösemi, Nilotinib, Tüberküloz

Conflict of Interest: The authors of this paper have no conflicts of interest, including specific financial interests, relationships, and/or affiliations relevant to the subject matter or materials included.

\section{References}

1. Druker BJ, Talpaz M, Resta DJ, Peng B, Buchdunger E, Ford JM, Lydon NB, Kantarjian H, Capdeville R, Ohno-Jones S, Sawyers CL. Efficacy and safety of a specific inhibitor of the BCR-ABL tyrosine kinase in chronic myeloid leukemia. N Engl J Med 2001;344:1031-1037.

2. Delomas T, Darne C, Besson C. Lack of recurrence of imatinib-induced interstitial lung disease with nilotinib. Leuk Lymphoma 2012;53:332-333. 
3. Dao K, Vedy D, Lopez J, Staneczek O, Buclin T, Livio F. Imatinib-induced dosedependent interstitial lung disease successfully switched to nilotinib: a case report with concentration exposure data. Int J Hematol 2013;97:299-300.

4. Lee NR, Jang JW, Kim HS, Yhim HY. Imatinib mesylate-induced interstitial lung disease in a patient with prior history of Mycobacterium tuberculosis infection. Korean J Intern Med 2015;30:550-553.

5. Eşkazan AE, Salihoğlu A, Erturan S, Soysal T. Interstitial pneumonitis in a patient with chronic myeloid leukemia. Turk J Hematol 2013;30:435-436.

6. Ohnishi K, Sakai F, Kudoh S, Ohno R. Twenty-seven cases of drug-induced interstitial lung disease associated with imatinib mesylate. Leukemia 2006;20:1162-1164.

7. Bergeron A, Rea D, Levy V, Picard C, Meignin V, Tamburini J, BruzzoniGiovanelli H, Calvo F, Tazi A, Rousselot P. Lung abnormalities after dasatinib treatment for chronic myeloid leukemia: a case series. Am J Respir Crit Care Med 2007;176:814-818.

8. Ando M, Okamoto I, Yamamoto N, Takeda K, Tamura K, Seto T, Ariyoshi Y, Fukuoka M. Predictive factors for interstitial lung disease, antitumor response, and survival in non-small-cell lung cancer patients treated with gefitinib. J Clin Oncol 2006;24:2549-2556.

\title{
Prostate Involvement in a Patient with Follicular Lymphoma
}

\author{
Foliküler Lenfomalı Hastada Prostat Tutulumu
}

\author{
Seda Yılmaz ${ }^{1}$, Sinan Demircioğlu ${ }^{1}$, Özlen Bektaş ${ }^{1}$, Özcan Çeneli ${ }^{1}$, Sıdıka Fındık ${ }^{2}$ \\ ${ }^{1}$ Necmettin Erbakan University Meram Medicine Faculty, Department of Hematology, Konya, Turkey \\ ${ }^{2}$ Necmettin Erbakan University Meram Medicine Faculty, Department of Pathology, Konya, Turkey
}

\section{To the Editor,}

While extranodal involvement is observed in $50 \%$ of cases of non-Hodgkin's lymphoma, prostatic involvement is rare. Prostatic lymphoma accounts for $0.09 \%$ of all prostate neoplasms and $0.1 \%$ of all non-Hodgkin's lymphomas [1].

Our patient was monitored for 4 years and had stage 4BS follicular lymphoma (bone marrow involvement; mesenteric lymph nodes in the abdomen, the largest of which was measured as $9 \times 4 \mathrm{~cm}$; cervical and mediastinal lymph nodes; and splenomegaly and B symptoms) at the time of diagnosis. He received CVP (cyclophosphamide, vincristine, prednisolone), CHOP (cyclophosphamide, adriamycin, vincristine, prednisolone), and gemcitabine therapy, respectively, and had lower urinary tract symptoms during follow-up. A hypertrophic prostate was palpated during the physical examination. The prostate-specific antigen (PSA) level was measured to be 8.3 (normal range: $0-4) \mathrm{ng} / \mathrm{mL}$. Urinary analysis showed microscopic hematuria. Ultrasound examination detected a prostate volume of $60 \mathrm{~mL}$. Transurethral resection of the prostate (TUR-P) pathology results showed a diffuse lymphocytic infiltration and positive staining for CD20, CD10, CD5, and BCL-2 (Figure 1). The symptoms of the patient regressed after treatment with rituximab plus bendamustine.

Prostate cancer is the most common cancer among men worldwide. There were $1,618,000$ cases with 366,000 deaths in 2015 [2]. Prostatic lymphoma is a rare condition that accounts for $0.09 \%$ of all prostate neoplasms. While extranodal involvement

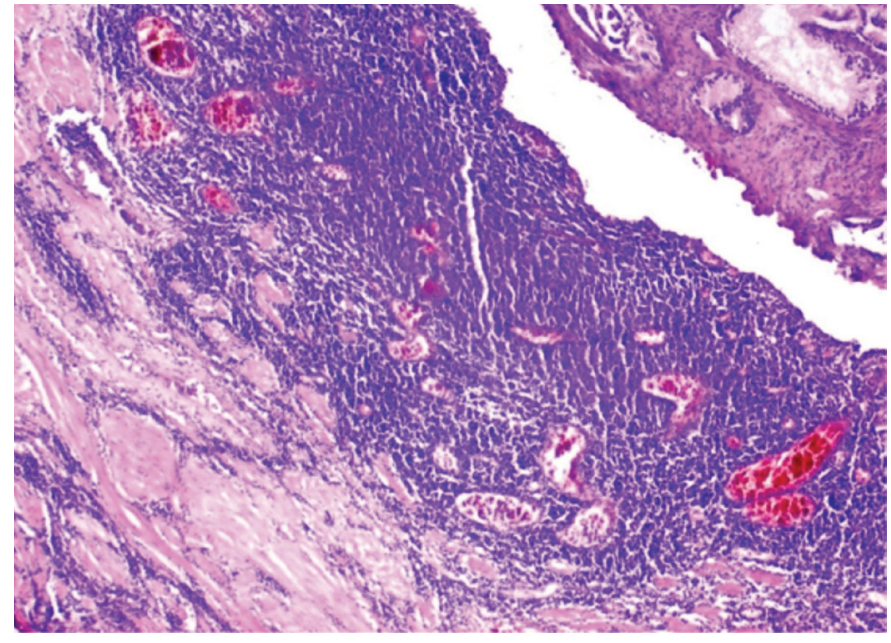

Figure 1. Diffuse lymphocytic infiltration.

is observed in about 50\% of cases of non-Hodgkin's lymphoma, prostatic involvement is rare. The usual clinical manifestations of prostatic involvement in lymphomas are lower urinary tract symptoms and acute urinary retention. High serum PSA levels are not typical for prostatic lymphoma. Our patient presented with high PSA levels.

A study that investigated prostate materials from 4831 subjects determined lymphoma in 29 subjects $(0.6 \%)$. Eleven $(0.23 \%)$ subjects had a history of concurrent lymphoma [3]. In patients with prostate cancer, the incidence of non-Hodgkin's lymphoma of the prostate was observed to be $0.2 \%$ in a series of 4319 radical prostatectomy cases [4] and $1.19 \%$ in another series of 1092 cases [5]. 